\title{
Performance Evaluation of WiMAX Networks through Large Scale Fading Channel Conditions
}

\author{
Priyanka \\ Department of Electronics \\ Ramgarhia Institute of Engineering and Technology \\ Phagwara, India
}

\author{
Jyoteesh Malhotra \\ Department of Electronics \\ G.N.D.U-Regional Campus, Jalandhar, India
}

\begin{abstract}
WiMAX is an effective metropolitan area broadband access technology, supplying wireless coverage over an area of several kilometers with high data rates. WiMAX technology has introduced WiMAX broadband to provide an alternative to the high cost of broadband connection through cable or DSL. In this paper, simulative investigations have been done for the WiMAX network using different path loss models. Investigations have been done in terms of performance metrics such as path loss, throughput and delay. Considering VoIP as major application, extensive results of different path loss models have been indicated which will help the network engineers in planning and design.
\end{abstract}

\section{General Terms}

WiMAX network, Path loss models.

\section{Keywords}

WiMAX, VoIP, OPNET, Path loss.

\section{INTRODUCTION}

Wireless networking has become an essential part in the modern telecommunication system. Hence, new ways to enhance the quality and speed of connectivity are being searched for. The demand of high speed data transfer with quality has led to the evolution of technology WiMAX (Worldwide Interoperability for Microwave Access) [1].

WiMAX is the leading standardized broadband wireless access technology based on the IEEE 802.16 [2] specifications. The standard was designed to provide 30 to 40 megabit-per-second data rates, with current rates providing up to $1 \mathrm{Gbit} / \mathrm{s}$ for fixed stations. It was defined to fill in the last mile, long range and to offer QoS and to support backhaul connectivity. WiMAX provides wide range of applications such as voice over Internet Protocol (VoIP), Internet Protocol Television (IPTV), mobile data TV, mobile emergency response services, and wireless backhaul as substitute for fiber optic cable [3].

As with any new technology, there are many issues that affect the implementation and utilization of WiMAX. The path of propagation being wireless, the performance of the network is affected by the environmental conditions of the area where the network is deployed. Keeping in mind the harsh wireless environment, investigations have been done in this work under varying terrain and environmental conditions.

The rest of the paper is organized as follows: Section II describes the related work. Section III provides about simulation methodology. Results are discussed in Section IV, before we finally conclude in section V.

\section{RELATED WORK}

There have been recent studies focusing on the performance evaluation of WiMAX networks.

The authors in [4] have analyzed the VoIP performance among the networks like WiMAX, WLAN and its integrated network in their paper. Performance evaluation is based on the parameters such as jitter, MOS, packet end to end delay. A comparative study for the three different network models has also been made for fixed and mobile users. It is observed that the WiMAX-WLAN integrated network provides improved and optimal performance over WLAN and WiMAX network with respect to network capacity and Quality of Service.

Another manuscript [5] reports the location based performance of WiMAX network with optimal base station. Performance is evaluated for the scenarios with different number of stationary and mobile nodes. The research helps for the deployment of WiMAX system to any country with good QoS. The results show that QoS has affected with the applications, voice over IP and MPEG.

A lot of information about WiMAX network can be found in literature [6]. The authors have studied the effect of base frequency on the performance of WiMAX and shown the effect of base frequency on MOS, packet end to end delay and throughput with G.711 as voice codec. The study also includes the influence of distance between BS and SS on the parameter path loss. They conclude that the best performance can be achieved at lower base frequencies.

The authors in [7] use OPNET modeler to simulate and compare the performance of Wi-Fi and WiMAX network in terms of mobility. Simulation results indicate that WiMAX may carry larger load and has better throughput. It also shows that the WiMAX queuing delay is smaller because of its broadband services while it is identical for two Wi-Fi scenarios i.e. one for stationary SS and other for mobile SSs.

\section{SIMULATION SETUP}

To evaluate the performance of the WiMAX network, a scenario was designed in the network simulator OPNET [8] with the assumption that the traffic generated in this network model is VoIP only. There are only peer-to-peer voice calls throughout the simulation, which means there is no voice conferencing and the subscriber stations (SS) are considered as fixed during the simulation runs. 


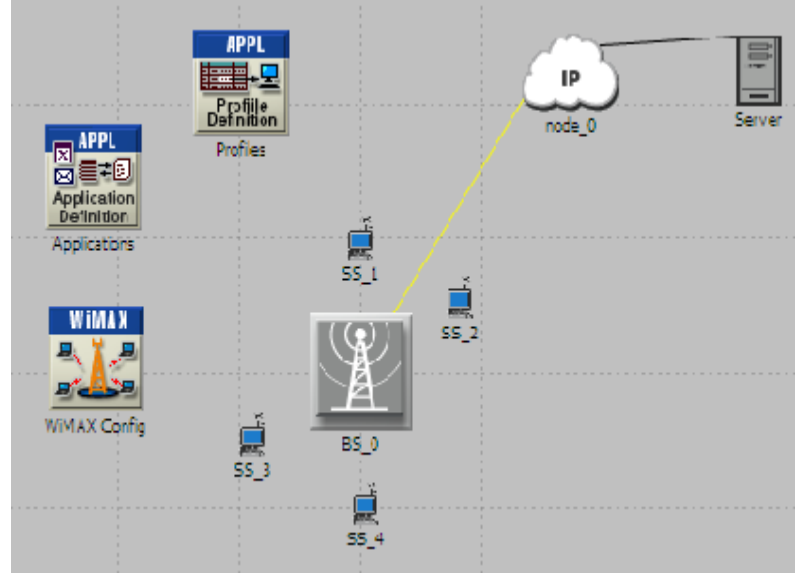

Fig.1 WiMAX Network Model

Fig. 1 illustrates the WiMAX network model considered in the simulations. The WiMAX network consists of four SSs and one base station (BS) which is connected to the server backbone having only one voice server through an IP backbone. The distance between the SS and BS is set to 1 Kilometers.

The WiMAX parameters used in the network are listed in the Table 1 \& Table 2

Table1. Network Design Parameters

\begin{tabular}{|c|c|}
\hline WiMAX Physical Profile & Wireless OFDMA 20MHz \\
\hline Bandwidth & 20MHz \\
\hline FFT & TDD \\
\hline Duplexing Technique & Physical Layer Enabled \\
\hline Efficiency Mode & ertPS \\
\hline WiMAX Service Class & $0.5 \mathrm{~W}$ \\
\hline BS Transmission Power & 16 QAM 3/4 \\
\hline SS Transmission Power & ITU Pedestrian B \\
\hline Modulation \& coding & Voice over IP \\
\hline Multipath Channel Model & G.711 \\
\hline Application & \\
\hline Voice codec &
\end{tabular}

\subsection{Path loss Model}

The common propagation path loss models are Free Space, Suburban Fixed (Erceg), Outdoor to Indoor and Pedestrian Environment and Vehicular Environment [1]. The Erceg's Suburban Fixed path loss model is further subdivided in three categories based on the building and tree density. These are summarized as,
Table2. Traffic Characteristics

\begin{tabular}{|c|c|}
\hline Match Property & IP ToS \\
\hline Match Condition & Equals \\
\hline Match Value & Interactive Voice \\
\hline
\end{tabular}

\subsubsection{Free Space Propagation model}

This environment is an ideal environment with transmitter and receiver has a clear line of sight path between them. No other sources of impairment and obstacles exist.

\subsubsection{Erceg's Suburban Fixed Model}

The Erceg model is based on extensive experimental data collected at $1.9 \mathrm{GHz}$ in 95 macro cells of suburban areas across the United States. The Erceg model is applicable mostly for fixed wireless deployment, with the MS installed under the eave/window or on the rooftop.

This path loss model is further subdivided in three categories.

\subsubsection{Terrain Type A}

This is a hilly terrain with moderate to heavy tree density, representing rural environments.

\subsubsection{Terrain Type B}

This is characterized by either a mostly flat terrain with moderate to heavy tree density or a hilly terrain with light tree density.

\subsubsection{Terrain Type $C$}

This is a flat terrain with light tree density for rural environments. This model is adopted by the IEEE 802.16 group as the recommended model for fixed broadband applications.

\subsubsection{Outdoor-to-Indoor and Pedestrian Path loss Model}

This environment is characterized by small cells and low transmission power. Base stations with low antenna heights are located outdoors; pedestrian users are located on streets and inside buildings and residences.

\subsubsection{Vehicular environment}

This environment is characterized by larger cells and higher transmits power.

\section{RESULTS AND DISCUSSION}

Environmental conditions play an important role in the network deployment. The performance of the WiMAX network is compared for free space, Outdoor to Indoor and Pedestrian environment, vehicular environment and three terrain types of Erceg path loss model in terms of performance metrics such as throughput, delay and path loss.

\subsection{Path loss}

Path loss (or path attenuation) is the reduction in power density (attenuation) of an electromagnetic wave as it propagates through space. Fig 2 shows the comparative results for average Path loss (in $\mathrm{dB}$ ) for path loss models used in the scenario. 


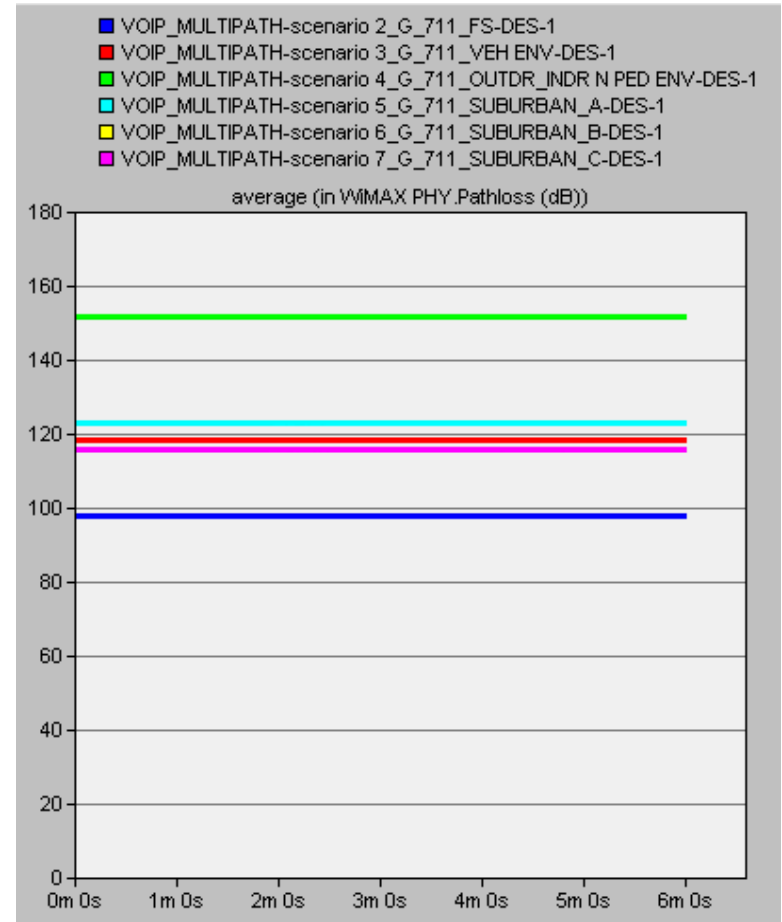

Fig.2 Path loss due to various path loss models in decibel

Path loss for outdoor to indoor and pedestrian is highest and for free space is lowest.

\subsection{Throughput}

Throughput represents the total data traffic in bits per second forwarded from WiMAX layer to higher layers in all WiMAX nodes of the network. Figure 3 shows the average throughput in bps for the path loss models used in the scenario.

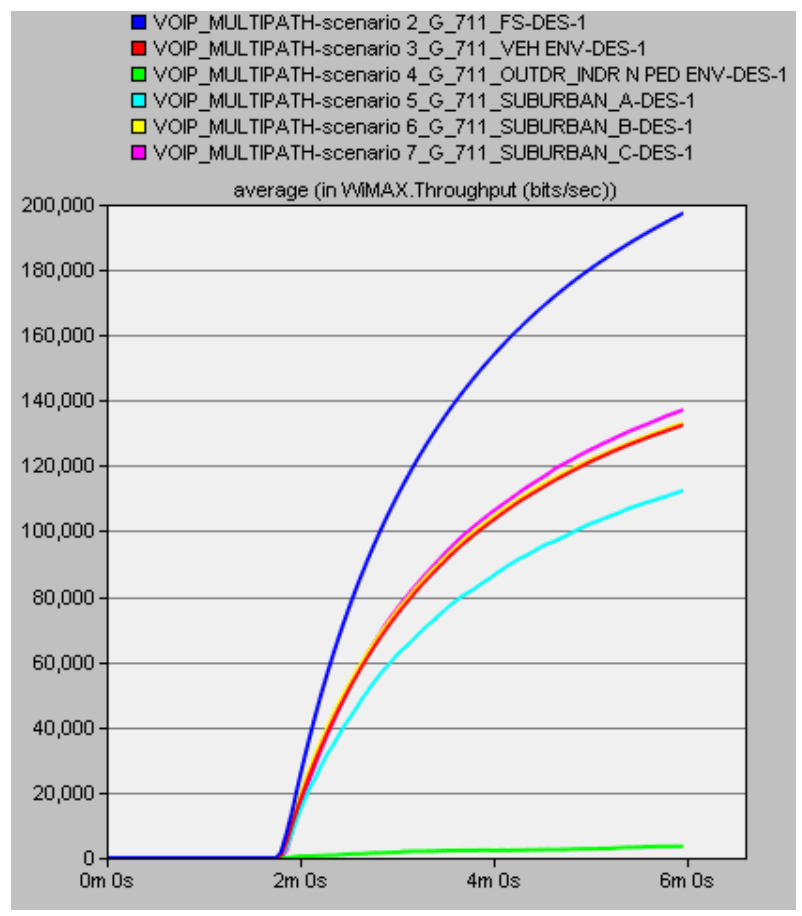

Fig.3 Throughput (bps)

Throughput of the network with free space path loss model is the highest and the same for Outdoor to indoor and pedestrian is the lowest.

\subsection{Delay}

Delay represents the end-to-end delay of all the packets received by the WiMAX MACs of all WiMAX nodes in the network and forwarded to the higher layer. Figure 4 shows the comparative results of average delay for different path loss models.

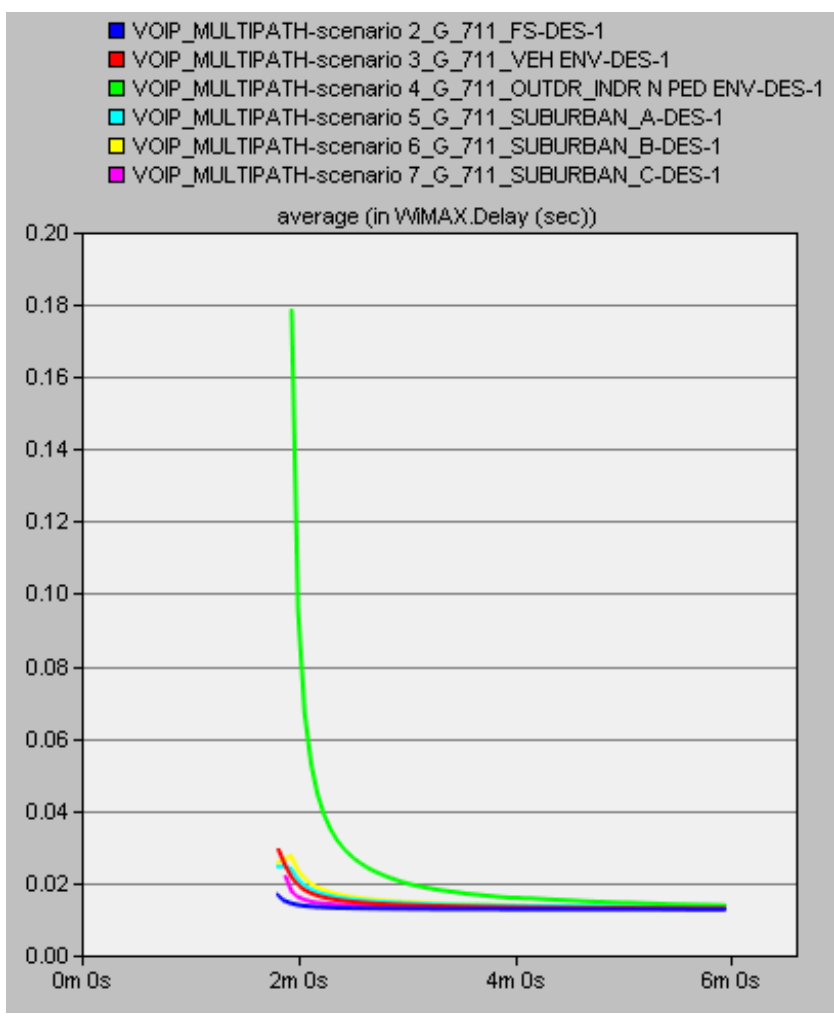

Fig.4 Average delay for different path loss models in WiMAX network

Delay of the network with free space as the path loss model is the lowest and the same for Outdoor to indoor and pedestrian is the highest.

The variation in path loss, delay and throughput are due to increased density of buildings, tree etc which results in enhanced reflections. For Outdoor to Indoor and Pedestrian environment, the number of reflections in the communicating path increases rapidly thereby causing huge path loss. Due to this, attenuation increases which in turn delay. This results in packet loss thereby causing fall in the throughput. For Free Space environment, impairments are less which results in less number of reflections and thus lowest path loss, lowest delay and highest throughput. For Erceg's suburban fixed models of terrain type A of hilly terrain with moderate to heavy tree density results in more reflections and thus highest path loss and delay among three terrain types which results in smallest throughput.

\subsection{Mean Opinion Score}

Mean Opinion Score is one of the most important performance metric used to measure the voice quality in VoIP. It gives a numerical indication of the perceived quality of the media received after being transmitted and eventually compressed using codecs. MOS is expressed in one number, from 1 to 5,1 being the worst and 5 the best. Figure 5 shows the comparative results of average MOS values for different path loss models used in the scenario. 


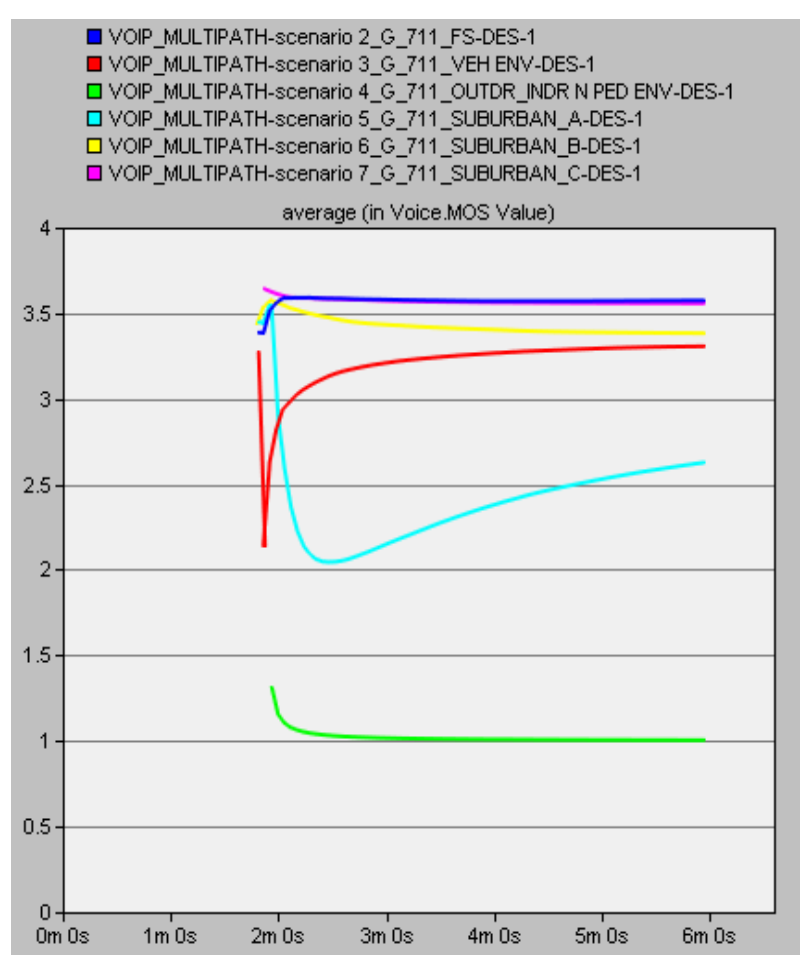

Fig.5 Average Mean Opinion Score for different path loss models

MOS depends upon number of packets dropped. For Outdoor to Indoor and Pedestrian environment, delay is maximum, which results in more packet loss and thus lowest value of mean opinion score. MOS of value 1 for Outdoor to Indoor and Pedestrian environment indicates poor voice quality. Voice having MOS of 3 can be of considerable quality. Hence free space path loss model shows good voice quality.

\section{CONCLUSION}

This paper shows the variation in the WiMAX network performance deployed over free space, Outdoor to Indoor and Pedestrian Environment, vehicular environment and suburban areas of terrain types varying from hilly terrain with moderate to heavy tree density to flat terrain with light tree density for Voice over IP application. The performance was evaluated on the basis of throughput, delay and path loss of WiMAX. As expected, the simulation results show that network performed best under free space environment which is an ideal environment. As environment change, density of obstacles changes which has severe effects on delay. As the number of obstacles increases, delay increases and throughput decreases.

\section{REFERENCES}

[1] Jeffrey G. Andrews, Ph.D, Arunabha Ghosh, Ph.D, "Fundamentals of WiMAX Understanding Broadband Wireless Networking," First Edition, Prentice Hall, 2007.

[2] IEEE Std 802.16 TM- 2004, "Part 16: Air Interface for fixed broadband wireless access systems" Oct 2004.

[3] Priyanka, Malhotra J. 2012, “ A Survey on PHY and MAC layer issues of future WiMAX networks" in the proceedings of UGC sponsored national seminar on Wireless Communication Networks.

[4] Kundu, A., Misra, I. S., Sanyal, S. K., Bhunia, S. "VoIP Performance over broadband wireless networks under static and mobile environments" International Journal of Wireless \& Mobile Networks (IJWMN) vol.2, no.4, pp. 82-93, Nov. 2010.

[5] Jha, R.K., Bholebawa, I.Z., Dalal, U.D. "Location based performance of WiMAX network for QoS with optimal BS" Wireless Engineering and Technology, vol.2, pp. 135-145, 2011.

[6] Gumaidah, B.F., Soliman, H. H. and Obayya, M. "Study the effect of Base Frequency on the performance of WiMAX network carrying voice" International Journal of computer Networks \& communications (IJCNC), vol.4, no.4, pp. 77-88, July 2012.

[7] Paul, R., Lally, S., Trajko vic, L., "simulation and Performance Evaluation of Wi-Fi and WiMAX using OPNET" OPNETWORK 2011, Washington, DC, Aug. 2011.

[8] OPNET Modeler, http://www.opnet.com/solutions/network_rd/modeler.ht $\mathrm{ml}$ 